Vol. 10(9), pp. 318-323, 16 May, 2015

DOI: $10.5897 /$ IJPS2015.4261

Article Number: 3C39B9D52942

ISSN 1992 - 1950

Copyright (C2015

International Journal of Physical

Author(s) retain the copyright of this article

Sciences

http://www.academicjournals.org/IJPS

\title{
Study on laser etching mechanism of aluminum thin film on polyimide
}

\author{
Liu Xiao-Li, Xiong Yu-Qing*, Ren Ni, Yang Jian-Ping, Wang Rui, Wu Gan and Wu Sheng-Hu \\ Science and Technology on Vacuum Technology and Physics Laboratory, Lanzhou Institute of Physics, Lanzhou \\ 730000, China.
}

Received 20 January, 2015; Accepted 16 April, 2015

\begin{abstract}
In order to study the laser etching mechanism for aluminum thin film on polyimide substrate, the etching process was simulated by the finite element analysis software ANSYS, and etching profile was predicted. A theoretical model was established by comparing the simulated etching results with calculated ones; it was presumed that the etching process was firstly a thermal dominant one, then a photochemical interaction dominant one, and finally a thermal one again.
\end{abstract}

Key words: Laser etching, aluminum thin film, polyimide, etching profile.

\section{INTRODUCTION}

Based on the principle of high-power short-pulsed laser interaction with matters, laser etching was widely employed for micromachining of multi-layer thin films of metal and polymer (Liu et al., 2014; Hu et al., 2011). In this case, the absorption of laser energy occurs rapidly and only in a very thin layer of metal film on the surface, and the film is thus instantaneously evaporated and removed while the substrate is unaffected due to the precise "cool" etching process. Understanding the mechanism of laser etching is essential to predict etching result and for a better improvement of the etching quality and precision.

The mechanism of laser etching has been studied extensively, Shin et al. (2007) suggested that laser etching of multi-layer thin films of metal and polymer is a combination of photochemical evaporation and thermal melt expulsion (Srinivasan et al., 1986). Some researches investigate into the properties of laser removal of metal thin film on polymer samples was carried out theoretically and experimentally in order to understand the mechanism of laser etching (Zhang et al., 2010; Zhou et al., 2005).

In this paper, in order to predict the etching profile of laser etching for aluminum thin film on polyimide substrate and enhance the micro-processing accuracy and quality, a theoretical model, called the Srinivasan-Smrtic-Babu (SSB) model, was adopted, which takes both thermal and photochemical effects of the laser etching into consideration. Parameters for SSB model were obtained by fitting the experimental data from relevant literature (Yoon and Bang, 2005; Shin et al., 2007) and ANSYS software was employed to study the temperature distribution within aluminum thin film on polyimide (PI) substrate during $355 \mathrm{~nm}$ laser etching process. The operating parameters include a TEM00 Gaussian distribution laser beam, pulse repetition frequency of 20

*Corresponding author. E-mail: xiongyq@hotmail.com

Author(s) agree that this article remain permanently open access under the terms of the Creative Commons Attribution License 4.0 International License

PACS: 02.70.Dh, 52.38.Mf, 61.41+e, 68.60.Dv, 72.15.Eb. 
$\mathrm{kHz}$, pulse width of $100 \mathrm{~ns}$. By comparing the simulated etching results with predicted ones, the processing of 1aser energy absorption and thermal conduction were analyzed, and a mechanism of metallic film/ polyimide substrate interface separation while metallic film in solid state due to the polyimide material thermal decomposition was also proposed subsequently.

\section{THEORETICAL MODELS}

\section{A theoretical model for PI removal by laser etching}

Photochemical and thermal effects were considered for ablation of polyimide $(\mathrm{Pl}), H_{c}$ is the etching depth per laser pulse by the photochemical effect according to the assumption of Beer's law (Li et al., 2006), which can be expressed as follows:

$$
H_{r}=a_{\text {eff }}^{-1} \ln \left(\frac{s}{-}\right)
$$

Where $a_{\mathrm{eff}}^{-1}$ is the absorption coefficient $\left(\mathrm{cm}^{-1}\right), F$ is the laser fluence per pulse $\left(\mathrm{J} / \mathrm{cm}^{2}\right)$, and $F_{\text {th }}$ is the threshold fluence $\left(\mathrm{J} / \mathrm{cm}^{2}\right)$.

When processing $\mathrm{PI}$, the thermal effect is non-ignorable, the single pulse etching depth expressed by $H_{\text {}}$, from pseudo-zeroth order rate law:

$-\frac{\mathrm{u}}{\mathrm{v}}=\mathrm{kc} \Rightarrow-\int_{-\mathrm{n}}^{\omega} \underline{\mathrm{u}}=\mathrm{k} \int_{n}^{\mathrm{L}} \mathrm{dt}$

$H_{\mathrm{T}}=k_{0} \times \mathrm{e}^{-\mathrm{E} / \mathrm{RT}}$

Where $k_{0}$ is the effective frequency factor ( $\mu \mathrm{m} /$ pulse), $E$ is the activation energy $\left(\mathrm{kJ} / \mathrm{cm}^{3}\right), R$ is the gas constant $\left(371 \mathrm{~J} /\left(\mathrm{cm}^{3} \cdot \mathrm{K}\right)\right)$, and $T$ is the temperature averaged in some manner over the irradiated region and is controlled by the incident fluence and the photon absorption dynamics of the Pl. Temperature within the laser exposed region is both time and position dependent. In the absence of knowledge of the exact functional dependence, it is only possible to proceed phenomenologically by assuming a one dimensional heat flow. The fluence dependence of local temperature can be modeled by an expression similar to that proposed by Yung et al. (2000):

$T(x)=\frac{w_{\mathrm{eff}^{-}}}{T} \times \mathrm{e}^{a_{\mathrm{eff}}{ }^{x}}$

Where $\rho$ is density $\left(\mathrm{kg} / \mathrm{cm}^{3}\right), C_{\mathrm{P}}$ is heat capacity $(\mathrm{J} / \mathrm{kg} \cdot \mathrm{K})$, and $x$ is the etching depth $(\mathrm{cm})$.

Calculate the $T_{\text {avg }}$ (average temperature) from $x=0$ to $x=d_{p}$ :

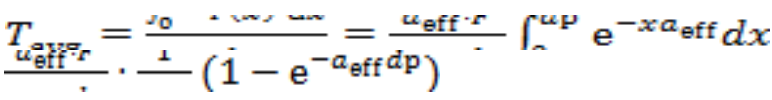

It can be simplified as:

$d \mathrm{p}=\stackrel{+}{ } \cdot \ln \frac{s}{m} \Longrightarrow d \mathrm{p} \cdot a_{\text {eff }}=\ln \frac{s}{m}$

By combining Equation (5) and (6), it can be obtained that:

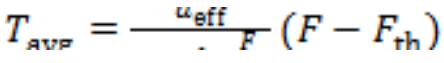

By putting Equation (7) into Equation (3), $H_{\top}$ (The etching depth by the photothermal effect) can be obtained as:

$H_{\mathrm{T}}=k_{0} \cdot \mathrm{e}^{\left\lfloor\left(-E^{*} \cdot \operatorname{In} F / F_{\text {th }}\right)\left(a_{\mathrm{eff}}\left(F-F_{\text {th }}\right)\right)\right\rfloor}$
Table 1. Parameters of PI for $355 \mathrm{~nm}$ UV laser etching.

\begin{tabular}{lc}
\hline Parameter & Values \\
\hline$F_{t h}\left(\mathrm{~J} / \mathrm{cm}^{2}\right)$ & 0.1 \\
$a_{\text {eff }}\left(\mathrm{cm}^{-1}\right)$ & $0.2 \times 10^{5}$ \\
$k_{0}(\mu \mathrm{m} /$ pulse $)$ & 8.86 \\
$E^{\star}\left(\mathrm{kJ} / \mathrm{cm}^{3}\right)$ & $207.6 \times 10^{3}$ \\
\hline
\end{tabular}

Where $E^{*}=\frac{{ }^{-}}{-}$is the effective activation energy $\left(\mathrm{kJ} / \mathrm{cm}^{3}\right)$, when photochemical and thermal effects were take into consideration, the etching depth per laser pulse, $H_{P I}$ can be expressed as follows:

$H_{\mathrm{DT}}=\stackrel{+}{x} \cdot \ln \frac{s}{r}+k_{\mathrm{n}} \cdot \mathrm{e}^{\left[\left(-E^{*} \cdot \operatorname{In} F / F_{\mathrm{th}} / /\left(\mathrm{a}_{\mathrm{eff}}\left(F-F_{\mathrm{th}}\right)\right]\right.\right.}$

By putting parameters in Table 1 into Equation (9), theoretical relationship between etching depth and fluence can be obtained, as shown in Figure 1.

In Figure 1, which shows the theoretical results in logarithmic scale, the etching depth will maintain at a constant value as the laser fluence increases. When the laser fluence is smaller than $1 \mathrm{~J} / \mathrm{cm}^{2}$, while photochemical effect is dominant, the etching depth as a logarithmic function increases rapidly, and then stays at a constant value. Reversely, the etching depth by thermal effect increases dramatically in the region of laser fluence greater than $5 \mathrm{~J} / \mathrm{cm}^{2}$. Although the etching depth is an exponential function of laser fluence, the logarithmic function in the exponent reduces the etching rate rapidly in the region of fluence greater than $25 \mathrm{~J} / \mathrm{cm}^{2}$. This indicates that a laser fluence greater than a certain value contributes little to the etching depth. Thus, it is meaningless to increase the laser fluence above this value.

\section{A theoretical model for Al removal by laser etching}

Considering that thermal effect is dominant during laser etching process of $\mathrm{Al}$, we adopt a theoretical model which takes only thermal effects into account, to predict the etching depth of laser etching of $\mathrm{Al}$ films. $H_{\mathrm{AL}}$ can be expressed as follows:

$H_{\mathrm{AI} \cdot}=H_{\mathrm{T}}=k_{\mathrm{n}} \cdot \mathrm{e}^{\left[\left(-E \cdot \ln F / F_{\mathrm{th}}\right) /\left(a_{\mathrm{eff}}\left(F-F_{\mathrm{th}}\right)\right]\right.}$

By putting parameters of $\mathrm{Al}$ in Table 2 into Equation (10), theoretical relationship between etching depth and laser fluence can be obtained, as shown in Figure 2. Figure 2 shows the theoretical results of etching depth considering only thermal effect in decimal scale. In the region of fluence smaller than $3.2 \mathrm{~J} / \mathrm{cm}^{2}$, the etching depth is not measurable; and in the region of fluence greater than 4 $\mathrm{J} / \mathrm{cm}^{2}$, the etching depth increases rapidly.

\section{SIMULATION SOLUTION}

Here, the adopted equations for laser-material interaction will be presented. Non-steady-state temperature distribution within thin film and substrate can be obtained by applying heat transfer and phase change model of laser heating. Ignoring gas and liquid dynamic effects $(\mathrm{Li}$ et al., 2008), differential equation describing temperature $T(x, y, t)$ of this process is:

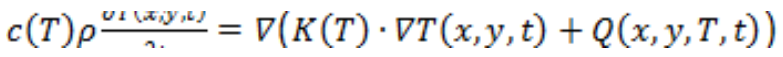




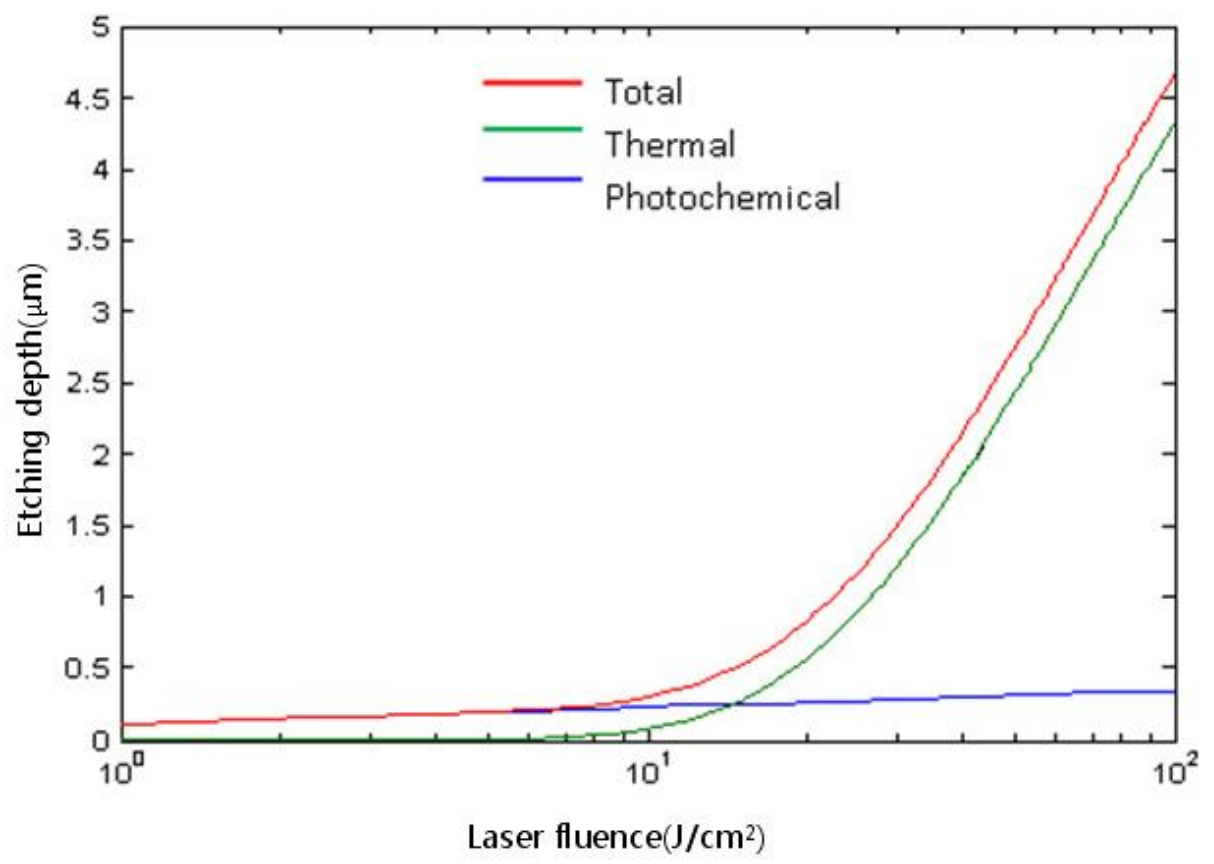

Figure 1. Relationship between etching depth and laser fluence for PI etched by $355 \mathrm{~nm}$ UV laser.

Table 2. Parameters of Al for $355 \mathrm{~nm}$ UV laser etching.

\begin{tabular}{lc}
\hline Parameter & Values \\
\hline$F_{\text {th }}\left(\mathrm{J} / \mathrm{cm}^{2}\right)$ & 0.32 \\
$a_{\text {eff }}\left(\mathrm{cm}^{-1}\right)$ & $5.917 \times 10^{5}$ \\
$k_{0}(\mu \mathrm{m} /$ pulse $)$ & 78 \\
$E^{*}\left(\mathrm{~kJ} / \mathrm{cm}^{3}\right)$ & $1.13 \times 10^{7}$ \\
\hline
\end{tabular}

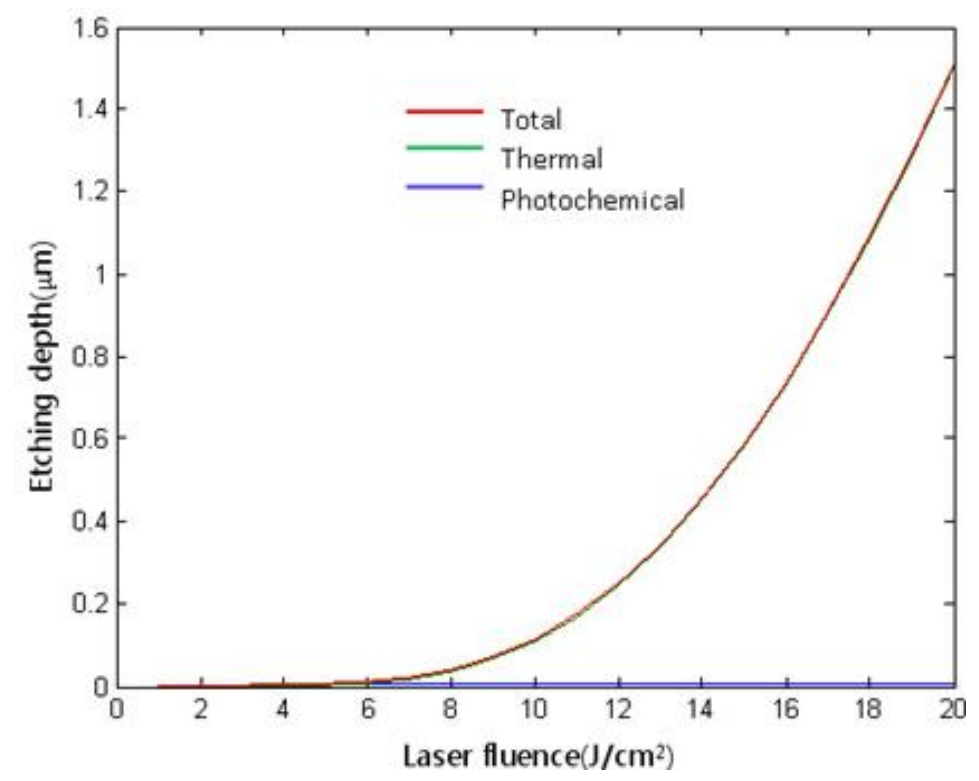

Figure 2. Relationship between etching depth and laser fluence for Al etched by $355 \mathrm{~nm}$ UV laser. 


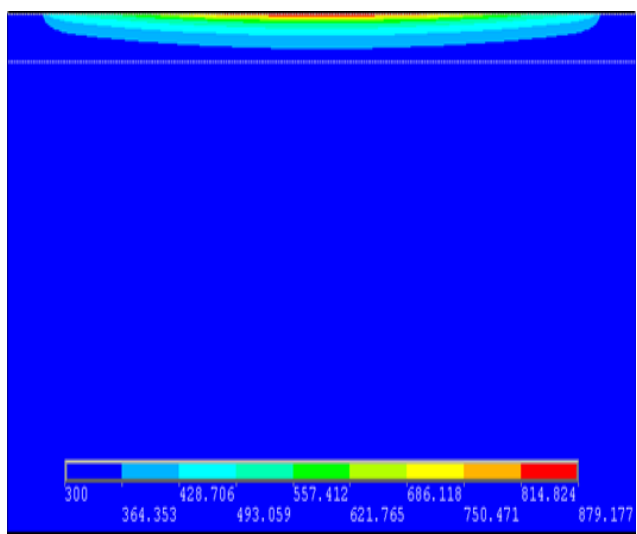

(a) $t=5 n s$

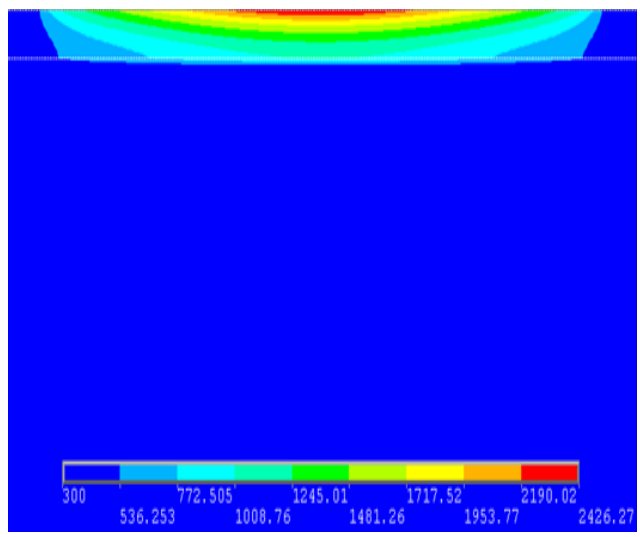

(c) $t=35 n s$

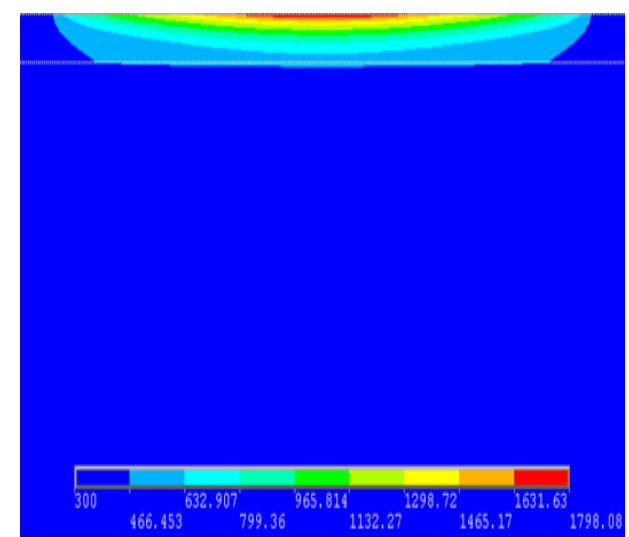

(b)t=20ns

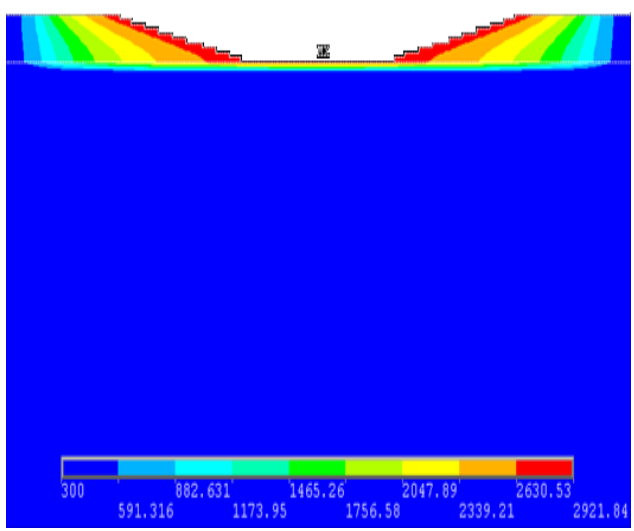

(d) $\mathrm{t}=100 \mathrm{~ns}$

Figure 3. Simulated temperature field caused by laser irradiation with different times (Different color represents different temperature).

Where $c$ is specific heat capacity, $\rho$ is material density, $K$ is thermal conductivity, and $Q$ is the heat generation rate. The initial temperature before laser irradiation equals to environmental temperature:

$T(x, y, 0)=T_{\text {enw }}$

The boundary conditions for temperature of the heated top surface and the other surfaces are different. Because of the high heat flow gradients during laser heating, no heat is lost from the heated side. Therefore, the Neumann boundary condition was used for boundary at $y=0$ (Hu and Yin, 1997). However, as the laser affected depth is much smaller than thickness of thin film, the opposite surface is assumed to stay at environmental temperature according to the Dirichlet boundary condition, expressed as follows:

$\left.\frac{\partial T(x, y, t)}{\partial z}\right|_{z=0}=0$

$\left.T(x, y, t)\right|_{z=\text { thickness of sample }}=T_{e n v}$
According to the theory of heat transfer in solid, analytical solution to Equation (11) can be obtained when laser power density is independent upon time. However, as one laser pulse generally offers several times the amount of energy required for one unit mass to rise from room temperature to evaporation temperature of $\mathrm{Al}$ within a very short period of time, the large heat flow gradient makes thermal properties of $\mathrm{Al}$ thin film temperature-dependent. Therefore, finite element method implemented in ANSYS was used to obtain the approximate numerical solution of the nonlinear model. This paper is based on theoretical and simulative analysis. The process of laser removal of Al thin film on PI samples was studied and the mechanism of the laser etching was analyzed.

\section{Numerical simulation for process of etching of aluminum thin film on PI}

Before simulation, the constant temperature boundary was assumed $\left(T_{\text {int }}=300 \mathrm{~K}\right)$. Figure 3 shows the temperature distribution during the laser etching of $\mathrm{Al} / \mathrm{PI}$ with different irradiation pulse. Figure 3(a) displays 


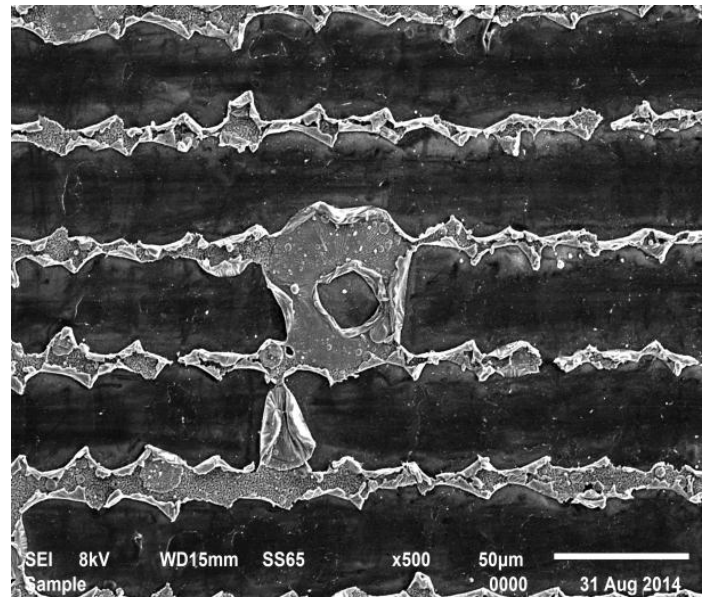

(a)

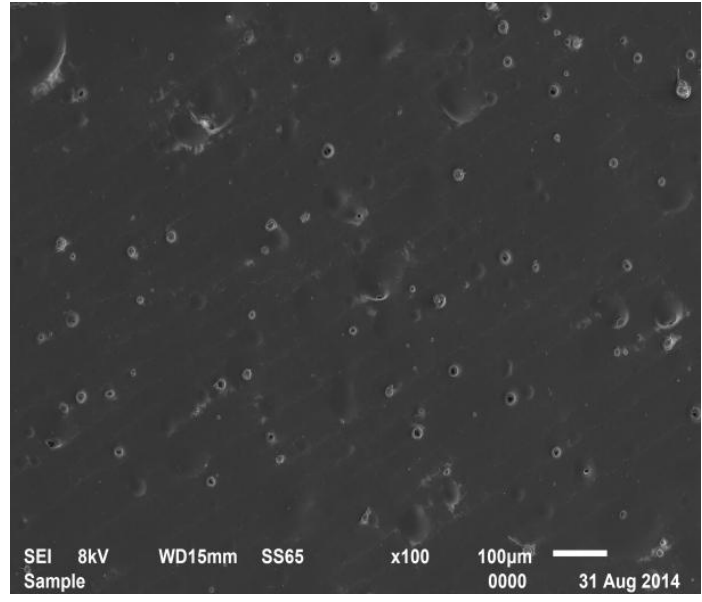

(b)

Figure 4. SEM images of etched morphology with different irradiation pulse.

temperature distribution within $\mathrm{Al} / \mathrm{PI}$ with a laser output power of $4 \mathrm{~W}$ and an irradiation pulse of $5 \mathrm{~ns}$. It can be seen that the maximum center temperature on the surface is $814.824 \mathrm{~K}$ and the interface temperature retains $300 \mathrm{~K}$, which means that the heat does not transfer to the substrate, and laser etching is a thermal process, for which the excitation energy is instantaneously transformed into heat. Over a period of time, as shown in Figure 3(b), temperature at interface reaches $466.453 \mathrm{~K}$, which is lower than the substrate's decomposing temperature, 500 $\mathrm{K}$. Figure 3(c) shows temperature distribution within Al/PI for a pulse of $35 \mathrm{~ns}$, it can be seen that the maximum temperature at the interface reaches about $772.505 \mathrm{~K}$, which is higher than substrate's decomposing temperature, which can result in substrate decomposition, and the pressure formed cause a state of separation between $\mathrm{Al}$ films and PI substrate. Meanwhile, heat conduction was blocked and the total energy is transformed to heat, induce rapid evaporation of Al films. As shown in Figure $3(d)$, it can be found that with the laser irradiation pulse change from $5 \mathrm{~ns}$ to $100 \mathrm{~ns}$, the surface temperature increases gradually and Al thin film was removed rapidly.

Experimental etching of Al thin film on PI was conducted to verify the simulated results. It shown that the etched $\mathrm{Al}$ thin film presents different edges with different irradiation pulse, corresponded to different laser fluence. The coarse edge of etched thin film in Figure 4(a) indicate that it was peeled off by pressure formed by decomposition of PI, and Figure 4(b) shows the bubbles formed on surface of PI caused by decomposition clearly.

\section{CONCLUSION}

By comparing of the simulated and calculated etching results, it can be concluded that the etching process was firstly a thermal interaction dominant one, aluminum thin film absorbs portion of the laser energy and transferred it into heat; then, when the temperature raised to decomposing temperature of polyimide, a photochemical interaction was dominant and polyimide at interface decomposed, a pressure is formed above the interface and peeled aluminum thin film off from polyimide substrate; Finally, temperature of floated aluminum increased and gasified, thermal mechanism is determinant again at this stage. The simulation was verified by experiment, and the simulated results can be used to optimize the etching parameters to obtain a smooth etching edge.

\section{Conflict of Interest}

The authors have not declared any conflict of interest.

\section{ACKNOWLEDGEMENT}

This work is supported by National Natural Science Foundation of China (NSFC. 51135005)

\section{REFERENCES}

Liu TH, Hao ZQ, Gao X, Liu ZH, Lin JQ (2014). Shadowgraph investigation of plasma shock wave evolution from Al target under 355-nm laser ablation. Chinese Phys. B 23(8):085203.

Hu HF, Ji Y, Hu Y, Ding XY, Liu XW, Guo JH, Wang XL, Zhai HC (2011). Thermal analysis of intense femtosecond laser ablation of aluminum. Chinese Phys. B. 20(4):044204.

Srinivasan V, Smrtic MA, Babu SV (1986). Excimer laser etching of polymers. J. App. Phys. 59(11):3861-3867.

Zhang F, Duan J, Zeng XY, LiXY (2010). $355 \mathrm{~nm}$ DPSS UV 
Laser Micro-Processing For Semiconductor and Electronics Industry, Proc. SPIE. 7584:75840-75850.

Zhou XL, Wen W, Zhang JS, Sun DB (2005). A mathematical model of the removal of gold thin film on polymer surface by laser ablation. Surf. Coat. Tech. 190:260-263.

Yoon KK, Bang SY (2005). Modeling of polymer ablation with excimer lasers. J. Korean Soc. Precision Eng. 22(9):60-68.

Shin BS, Oh JY, Sohn H (2007). Theoretical and experimental investigations into laser ablation of polyimide and copper films with 355-nm Nd: $\mathrm{YVO}_{4}$ laser. J. Mater. Process. Tech. 187-188:260-263.

Li L, Zhang D, Li Z, Guan L, Tan X, Fang R, Hu D, Liu G (2006). The investigation of optical characteristics of metal target in high power laser ablation. Physica B. 383(2):262-266.

Yung WKC, Liu JS, Man HC, Yue TM (2000). 355 nm Nd:YAG laser ablation of polyimide and its thermal effect. J. Mater. Process. Technol. 101:306 311.
Li J, Peterson GP, Cheng P (2008). Dynamic characteristics of transient boiling on a square platinum microheater under millisecond pulsed heating. Int. J. Heat Mass Tran. 51:273-282.

Hu B, Yin HM (1997). On Critical Exponents for the Heat Equation with a Mixed Nonlinear Dirichlet-Neumann Boundary Condition. J. Math. Anal. Appl. 209:683-711. 